\title{
Les avancées et les revers de la révision constitutionnelle en outre-mer
}

Simple réajustement du corset juridique ou esquisse d'un desserrement progressif?

The review of the Constitution and its breakthroughs and setbacks in the Overseas territories: simply a re-adjustment of the "legal corset" or the beginnings of a gradual and future letting-go?

\section{Nadège Damoiseau}

\section{(2) OpenEdition}

\section{Journals}

Édition électronique

URL : http://journals.openedition.org/plc/115

DOI : $10.4000 /$ plc. 115

ISSN : $2117-5209$

Éditeur

L'Harmattan

\section{Édition imprimée}

Date de publication : 15 décembre 2007

Pagination : 41-76

ISBN : 2-296-01986-2

ISSN : $1279-8657$

Référence électronique

Nadège Damoiseau, "Les avancées et les revers de la révision constitutionnelle en outre-mer », Pouvoirs dans la Caraïbe [En ligne], 15 | 2007, mis en ligne le 06 mai 2011, consulté le 14 novembre 2019. URL : http://journals.openedition.org/plc/115; DOI : 10.4000/plc.115 


\title{
LES AVANCEES ET LES REVERS DE LA REVISION CONSTITUTIONNELLE EN OUTRE-MER :

\author{
SIMPLE REAJUSTEMENT DU CORSET JURIDIQUE OU
} ESQUISSE D'UN DESSERREMENT PROGRESSIF ?
}

\author{
Nadège DAMOISEAU \\ Docteur en droit public, ancienne ATER \\ Université des Antilles et de la Guyane
}

La révision constitutionnelle du 28 mars 2003 a été une étape attendue par l'outre-mer ${ }^{1}$. Trop de critiques pesaient sur l'ancienne rédaction, à la fois incohérente, dépassée, rigide, contraignante, directrice, inadaptée, uniforme... Ces termes risquaient à la longue de ternir la réputation de la charte suprême.

Le Constituant a entendu remédier à cela et faire en sorte que les aspirations locales ne soient plus étouffées. Il en résulte un nouveau cadre qui conjugue les dispositions ayant trait à l'intégrité du territoire et à l'indivisibilité à des temps plus prometteurs pour la démocratie locale. En effet, la diversité et la souplesse ne sont plus des termes tabous et inspirent désormais la nouvelle architecture territoriale. Ils contribuent même à donner une portée juridique certaine au droit d'initiative encore balbutiant et à l'évolution statutaire enserrée jusqu'alors dans un corset juridique.

Cependant, cette nouvelle orientation suscite des craintes. Faut-il y voir un désengagement déguisé des autorités compétentes de la République vis-à-vis de l'outre-mer ainsi qu'un début de remise en cause de la logique de l'Etat unitaire? Non, si on considère le fait que plus les dispositions constitutionnelles propres à l'autonomie juridique gagnent du terrain, plus l'Etat réitère son encadrement juridique d'antan.

Pourtant, ces garanties ne suffisent pas à crédibiliser totalement les avancées du texte constitutionnel. Des inquiétudes surgissent qui relancent l'interrogation et altèrent ses premières applications concrètes. Elles trouvent leur source dans des éléments juridico-politiques. Les ambiguïtés du nouveau dispositif relatives aux modifications juridiques présentes et à venir n'ont pas fini de diviser la classe politique locale.

Qu'en est-il en réalité ? S'agit-il d'un simple réajustement du corset juridique ou de l'esquisse d'un desserrement progressif ? Il s'agira de percer

\footnotetext{
${ }^{1}$ Les notions d'outre-mer ou de DOM-TOM ont peu à peu cédé la place à celle d'outre-mers au pluriel.
} 
toute l'ambivalence de cette question, à travers l'étude d'une part des avancées du texte constitutionnel dans la nouvelle organisation décentralisée de l'Etat unitaire français (I), d'autre part de ses possibles zones d'ombre (II).

\section{LES AVANCEES DU TEXTE CONSTITUTIONNEL}

La Constitution révisée comprend toute une série de dispositions que l'on pourrait qualifier de communes, spécifiques, voire «ciblées $»^{2}$. Les premières s'appliquent à l'ensemble des collectivités territoriales de la République. Les secondes et les dernières visent spécialement chacune des catégories du nouveau binôme ultra-marin auquel il faut ajouter le cas à part de la Nouvelle-Calédonie. Cette précision étant faite, les premières traduisent un renforcement manifeste du poids des outre-mers au sein de la constitution (A), les autres, un nouveau classement plus souple (B).

\section{A - LE RENFORCEMENT DU POIDS DE L'OUTRE-MER AU SEIN DE LA CONSTITUTION}

Les outre-mers bénéficient tout d'abord d'un réajustement rédactionnel qui les vise spécifiquement. Les premières modifications concernent l'élection et le pouvoir de nomination du président de la République. En effet, l'article 7 de la Constitution prévoit si nécessaire ${ }^{3}$, et ce afin de remédier au décalage horaire qui démotivait les électeurs d'outre-mer, l'organisation d'un second tour intervenant quatorze jours après le premier. De plus, le pouvoir de nomination de l'article 13 est étendu aux représentants de l'Etat dans une nouvelle catégorie de collectivités territoriales, les collectivités d'outre-mer (C.O.M. $)^{4}$, et en Nouvelle-Calédonie ${ }^{5}$. Les liens de celles-ci avec la République française s'en trouvent déjà confortés. Puis, le Constituant modifie l'ordre de

\footnotetext{
${ }^{2}$ Il existe encore une subdivision au sein de la spécificité : notamment pour régir les collectivités dotées de l'autonomie.

${ }^{3} \mathrm{Si}$ la majorité absolue des suffrages exprimés n'est pas obtenue au premier tour de scrutin.

${ }^{4}$ Elles sont au nombre de quatre : Mayotte, Saint-Pierre et Miquelon, la Polynésie française et Wallis-et-Futuna.

${ }^{5}$ Pour la Nouvelle-Calédonie, il s'agit du haut-commissaire de la République : article 13, alinéa 3 modifié, et 72, alinéa 6 de la Constitution.
} 
priorité des chambres pour l'examen de certains projets de $1 \mathrm{i}^{6}$. Pour la première fois sous la $\mathrm{V}^{\circ}$ République, le Sénat risque d'influer sur l'organisation des collectivités territoriales. Celle des outre-mers en particulier.

Les outre-mers jouissent ensuite de dispositions nouvelles qui ont non seulement pour effet de maintenir leurs garanties mais aussi de les faire participer davantage aux décisions qui les concernent. Certaines ont trait à l'intégrité du territoire de la République, d'autres touchent plutôt à l'indivisibilité de l'exercice de la souveraineté sur ce dernier.

Parmi celles qui visent l'intégrité, commençons par relever que les outre-mers n'obéissent plus, la Nouvelle-Calédonie mise à part, à la division tripartite initiale : à savoir les collectivités ${ }^{7}$ à identité législative régies par l'article 73, celles à spécialité législative ${ }^{8}$ relevant de l'article 74, et celles sui generi $^{9}{ }^{9}$ créées par le législateur en application de l'article 72 . Le Constituant a souhaité ce classement trop rigide pour une division binaire plus souple où la diversité $^{10}$ n'est plus nécessairement source d'autonomie ou d'indépendance. Désormais, il y a d'une part les DOM/ROM ${ }^{11}$ et les collectivités qui pourraient se substituer à eux relevant de l'article 73, d'autre part les C.O.M. régies par l'article 74. Chacune des collectivités qui les composent a clairement sa place au sein d'une liste ${ }^{12}$. Cet ancrage ${ }^{13}$ devrait commencer à rassurer certaines populations d'outre-mer ${ }^{14}$, voire certains élus politiques ${ }^{15}$ face au spectre de la perte du statut convoité ou acquis.

\footnotetext{
${ }^{6}$ Selon l'alinéa modifié de l'article 39, « sans préjudice du premier alinéa de l'article 44, les projets de loi ayant pour principal objet l'organisation des collectivités territoriales et les projets de loi relatifs aux instances représentatives des Français établis hors de France sont soumis en premier lieu au Sénat».

${ }^{7}$ Les quatre D.O.M. et les quatre R.O.M.

${ }^{8}$ Les trois T.O.M. (Polynésie française, Wallis-et-Futuna et les Terres australes et antarctiques françaises).

${ }^{9}$ Mayotte et Saint-Pierre-et-Miquelon.

${ }^{10}$ Ce terme suit la ligne déjà projetée par Monsieur Jacques Chirac le 18 mai 2001 à SaintDenis. Voir R. Bacque, «M. Chirac propose une révision constitutionnelle pour offrir des statuts à la carte 'à l'outre-mer' », Le Monde, dimanche 20-lundi 21 mai 2001, p.7.

${ }^{11}$ Les départements, les régions d'outre-mer (Guadeloupe, Guyane, Martinique et Réunion).

${ }^{12}$ Les collectivités ultra-marines sont mentionnées nominativement à l'article 72.3 de la Constitution.

${ }^{13}$ La réforme confirme le fait que les collectivités constituent des parties d'un même territoire, celui de la République Française. Sur la notion «d'ancrage», voir M. Verpeaux, «La République, la Constitution de 1958 et le Conseil constitutionnel », dans la République, Mélanges Pierre Avril, Paris, Montchrestien, 2001, 632 p., pp. 129-139.

${ }^{14}$ En particulier, la population mahoraise.
} 
Concernant les collectivités de la première catégorie, outre le maintien du principe d'application de plein droit du droit métropolitain, il est toujours possible de prévoir des adaptations. Celles-ci bénéficient même d'un champ d'application un peu plus souple ${ }^{16}$ qui laisse entrevoir une meilleure prise en compte des spécificités des D.O.M. et donc un desserrement léger d'un des verrous constitutionnels ${ }^{17}$. Seule La Réunion ${ }^{18}$ est et reste attachée à l'idée d'un statu quo absolu. Par là même, elle se distingue de la Guadeloupe, de la Martinique, et de la Guyane, plus favorables dans leur grande majorité ${ }^{19}$ à suivre les avancées du texte constitutionnel vers plus de démocratie locale.

Concernant les collectivités de la deuxième catégorie, elles se substituent aux ex-T.O.M. et aux collectivités sui generis. Mayotte et SaintPierre-et-Miquelon s'alignent désormais avec les Iles Wallis-et-Futuna et la Polynésie française et comme leurs nouvelles consœurs, verront la loi organique fixer leur statut respectif ${ }^{20}$. S'y ajoutent Saint-Barthélemy et SaintMartin, deux ex-communes de Guadeloupe. Précisons que ces deux nouvelles entités ont opté - à la suite des consultations populaires organisées dans la Caraïbe le 7 décembre 2003 - pour la transformation statutaire. L'exigence d'une mention expresse d'application des lois est toujours de rigueur au sein de l'article 74, mais de manière moins impérative ${ }^{21}$. En effet, il ne faudrait pas gêner une collectivité sur le chemin progressif de la départementalisation,

\footnotetext{
${ }^{15}$ Notamment les forces politiques de droite de La Réunion.

${ }^{16} \mathrm{Ce}$ terme de souplesse tend à remplacer celui de rigidité issu de l'ancienne rédaction.

${ }^{17} \mathrm{La}$ jurisprudence constitutionnelle avait jusqu'alors une interprétation restrictive quant aux capacités des D.O.M./R.O.M. à s'écarter de la loi.

${ }^{18}$ Les départementalistes réunionnais s'étaient déjà opposés à l'institution d'une «assemblée délibérante unique » dans les D.O.M. en 1982 et à la bi-départementalisation de La Réunion en 2000 ; A. Oraison, «Quelques réflexions générales sur l'article 73 de la Constitution de la $\mathrm{V}^{\mathrm{e}}$ République, corrigé et complété par la loi constitutionnelle du 28 mars 2003 (Les possibilités offertes aux départements d'outre-mer - Guadeloupe, Guyane, Martinique - en matière d'habilitation législative et l'exception insolite du département de La Réunion)», Revue Juridique et Politique, 2003 - n² 2, p. 242. De même, ils se sont montrés hostiles au projet de décentralisation de Jean-Pierre Raffarin ; voir article d'A. Oraison précité, p. 241.

${ }_{19}$ Seule la Guyane n'a pu obtenir un consensus politique suffisant pour l'organisation d'un référendum local.

${ }^{20}$ Il s'agit surtout d'une innovation importante pour Mayotte et Saint-Pierre-et-Miquelon.

${ }^{21}$ Le principe de spécialité devient modulable.
} 
c'est-à-dire en voie d'être entièrement régie par les règles du droit commun. Nous pensons à la collectivité départementale de Mayotte ${ }^{22}$.

En dehors de cette division binaire, la Nouvelle-Calédonie qui avait été oubliée dans le projet gouvernemental se voit réintégrée dans la «liste » parmi les collectivités à statut particulier. Son régime juridique relève toujours du titre XIII de la Constitution intitulé «Dispositions transitoires relatives à la Nouvelle-Calédonie ». Et elle continue à jouir avec la Polynésie française d'un statut d'autonomie défini et garanti pour partie par la Constitution ${ }^{23}$. Cela dit, pour évoquer leur qualification juridico-politique, Olivier Gohin parle non pas de fédéralisme mais de « régionalisme asymétrique ${ }^{24}$.

Certaines dispositions du titre XII de la Constitution visant aussi la Nouvelle-Calédonie témoignent même d'un resserrement du «corset juridique ». Citons notamment les alinéas 1 et 5 de l'article 72 qui incluent la collectivité particulière parmi les collectivités territoriales de la République, ce qui la place du même coup, tout comme les autres, sous l'empire du contrôle administratif. Ou encore et surtout l'article 74-1 qui permet au gouvernement de légiférer par voie d'ordonnances sans habilitation préalable du Parlement ${ }^{25}$. Certes, ce pouvoir d'action s'effectue dans les domaines relevant de la compétence de l'Etat, mais aussi et c'est ce qu'il faut retenir, dans les mêmes conditions qu'à l'égard des autres collectivités d'outre-mer. Faut-il y voir un nouveau moyen constitutionnel de passer outre certaines réticences à l'égard de la sécession, ou, au contraire un mode utile d'affermissement de la souveraineté en cas d'impasse du processus actuel ? Pour certains, il est loisible de le penser ${ }^{26}$. Ces opinions se justifient par la

\footnotetext{
${ }^{22}$ Il faudra que le Conseil constitutionnel amené à se pencher sur l'examen d'une loi organique relative à cette collectivité confirme le lien de rupture entre l'article 74 et le principe de spécialité législative. En ce sens et sur les conséquences complexes de l'application de l'article 74 pour Mayotte, voir J.-P., Thiellay, «Les outre-mers dans la réforme de la Constitution », AJDA 24 mars 2003, p. 569.

${ }^{23}$ Ces collectivités ont aussi un statut d'autonomie relevant d'une décentralisation politique qui ne concerne pas les autres collectivités territoriales.

${ }^{24} \mathrm{O}$. Gohin, «La nouvelle décentralisation et la réforme de l'Etat en France », AJDA, 24 mars 2003 , p. 526.

${ }^{25}$ Toutefois la loi en cause peut expressément exclure le recours à de telles ordonnances et surtout celles-ci deviennent caduques en l'absence de ratification par le Parlement à l'issue d'un délai de dix-huit mois suivant leur publication.

${ }^{26}$ J. Jorda, «Les collectivités territoriales outre-mer et la révision de la Constitution », Revue française du Droit constitutionnel, 56, 2003, p. 723.
} 
mainmise gouvernementale qui pourrait encore constituer une réalité juridique effective. Celles-ci ajoutent que cette éventualité n'est pas si anodine si l'on en juge par le faible engouement des électeurs corses à l'occasion de leur tout premier référendum.

Notons que dans le cadre de ce réajustement territorial, la région d'outre-mer est finalement reconnue ${ }^{27}$. De plus, le législateur peut créer une nouvelle collectivité territoriale le cas échéant en lieu et place d'une ou plusieurs autres ${ }^{28}$. Celle-ci se nommera à «statut propre» ou à «statut particulier » ou toute autre dénomination voulue par lui pour diversifier le cadre territorial. Encore une fois, la classification d'inspiration uniforme n'est à l'évidence plus de mise dans le nouveau texte constitutionnel.

En plus de ce «bouleversement ultramarin ${ }^{29} \mathrm{du}$ cadre territorial, l'ensemble de l'outre-mer est concerné par les dispositions communes de l'article 72-1 de la Constitution relatives au droit de pétition, au « référendum décisionnel » et aux consultations au niveau local. Chacun de ces procédés constitue une avancée pour la démocratie locale.

On connaît déjà la portée relative de la consultation locale. Rappelons en effet que lors de la présentation du projet de loi pour l'outre-mer, le gouvernement avait vite tracé les limites de la libre détermination statutaire ${ }^{30}$. Le Conseil constitutionnel ne l'avait pas contredit ${ }^{31}$ conférant ainsi à la consultation de la collectivité concernée la valeur de «simple avis ». La possibilité ouverte au législateur - selon l'alinéa 3 - de consulter ou pas les

\footnotetext{
${ }^{27}$ Voir la décision fondamentale de reconnaissance des régions d'outre-mer : C.C., décision $\mathrm{n}^{\circ}$ 84-174 DC du 25 juillet 1984, Compétence des régions d'outre-mer, Rec., p. 84 ; Egalement L. Touvet, J. Ferstenbert et C. Cornet, «Les grands arrêts du droit de la décentralisation »,

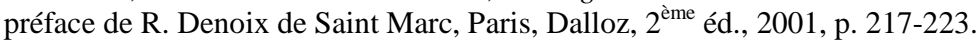

${ }^{28}$ Cette faculté introduite à l'article 72 sera reconduite pour l'outre-mer à l'article 73 dernier alinéa.

${ }^{29}$ Voir A. Oraison, art. prec., p. 239.

${ }^{30}$ Doc. AN., $\mathrm{n}^{\circ} 2322$; Le gouvernement avait précisé qu'il lui appartiendrait « de s'assurer du consentement des populations intéressées et une fois celui-ci vérifié, d'en tirer, de façon spécifique pour chacun des départements concernés, les conséquences juridiques qui en découleront ».

${ }^{31}$ Bien que la haute juridiction crée l'incompréhension en donnant à la procédure purement consultative la même base constitutionnelle que celle de ratification, elle consacre définitivement leur nette distinction en laissant aux autorités compétentes de la République toute liberté pour tenir compte ou pas du choix éventuellement émis par les collectivités territoriales d'outre-mer; $C f$. : Cons. 6 ( $1^{\text {ère }}$ et $3^{\text {ème }}$ partie) de la décision n ${ }^{\circ} 2000-428 \mathrm{DC}$ du 4 mai 2000, Consultation de la population de Mayotte.
} 
électeurs concernés ${ }^{32}$ dès lors qu'il décide de créer une collectivité territoriale à statut particulier ou de modifier son organisation ne devrait donc pas donner à cette éventuelle participation locale une portée juridique plus grande. De même concernant l'opportunité de consulter les électeurs en cas de modification des limites des collectivités territoriales contenue dans la dernière phrase de l'article 72-1. Pour autant, ne pas avoir une portée juridique importante ne veut pas dire aucune influence sur la décision finale. La prise en compte des aspirations locales est à l'honneur dans cette réforme et correspond d'ailleurs aux annonces déjà faites par le président de la République ${ }^{33}$. Aussi, par exemple, rien n'empêchait le gouvernement d'aller jusqu'au bout de son projet de «bi-départementalisation » de La Réunion en 2000, si ce n'est le défaut de consentement d'une majorité supposée dans l'île.

En revanche, l'introduction ouverte à l'alinéa 2 de l'article 72-1 du référendum local décisionnel pèse de tout son poids dans la balance de la démocratie participative directe des «populations d'outre-mer». La loi constitutionnelle - qui prévoit que "des projets de délibération ou d'actes relevant de la compétence d'une collectivité territoriale peuvent, à son initiative, être soumis, par la voie du référendum, à la décision des électeurs de cette collectivité » - fait enfin en sorte que le mécanisme de consultation aboutisse à une décision ${ }^{34}$. Certes, des ambiguités subsistent ${ }^{35}$ et les liens du «corset juridique » demeurent encore solides ${ }^{36}$ mais on peut dire qu'une des ficelles a commencé à se détendre et à esquisser la voie d'un desserrement progressif.

Le droit de pétition résultant de l'alinéa 1 du même article constitue lui aussi un renforcement de la démocratie directe locale. Il n'est pas sans lien avec les référendums et la consultation des électeurs puisqu'une fraction

\footnotetext{
${ }^{32}$ La définition des électeurs intéressés, qui n'est pas précisée par l'alinéa 3 mentionnant la consultation «des électeurs », devra être faite par la loi.

${ }^{33}$ Lors de ses discours sur l'avenir économique et institutionnel des départements d'outre-mer le 11 mars 2000 en Martinique et le 18 mai 2001 à La Réunion.

${ }^{34}$ M. Verpeaux, «Référendum local, Consultations locales et Constitution », AJDA, 24 mars 2003, p. 540.

${ }^{35}$ Pour Michel Verpeaux, beaucoup d'éléments restent à régler par la loi organique chargée de compléter l'article, notamment préciser les seuils de participation des électeurs nécessaires pour valider le résultat de la consultation ou encore prévoir des délais pour encadrer la nouvelle possibilité d'organisation référendaire ; op. cit., p. 543.

${ }^{36}$ Il n'est pas prévu que les actes locaux échappent au contrôle de légalité exercé par le représentant de l'Etat. Est même envisagé un contrôle de légalité «renforcé », comme un contrôle préalable du référendum par une sorte de référé pré-consultation ; op. cit.., p. 543.
} 
de la population d'une collectivité pourra elle-même demander à auprès de l'assemblée délibérante l'organisation de sa propre consultation sur une question de compétence propre ${ }^{37}$. L'action locale devient ainsi dans la nouvelle organisation décentralisée de l'Etat unitaire français une force impulsive et décisive pour l'outre-mer et les autres. Nous pensons tout particulièrement à la Corse exclue jusqu'à cette révision de toute retombée jurisprudentielle ultramarine ${ }^{38}$. En effet, rappelons de prime abord que cette dernière ne remplissait pas les conditions constitutionnelles pour bénéficier de la consultation d'autodétermination ouverte sur la base de l'article 53 alinéa 3 de la Constitution et réservée - si on se réfère à l'alinéa $2 \mathrm{du}$ Préambule - aux «territoires d'outre-mer ». Il s'agissait d'une lacune car la doctrine et la pratique politique avaient indéniablement donné valeur de « consentement » à la consultation populaire. Cette exclusion s'est d'ailleurs confirmée en 1991 lorsque la Haute Juridiction a déclaré « inconstitutionnelle» la référence au peuple corse. De plus, l'obstacle constitutionnel ne s'était pas limité à l'hypothèse de sécession, mais aussi à celle d'évolution statutaire. Ainsi, cette collectivité n'a pas eu l'opportunité de jouir du référendum consultatif même si la portée politique de celui-ci était moindre comme nous l'illustrerons plus loin. Ajoutons enfin que le juge constitutionnel avait confirmé une nouvelle fois ses limites territoriales lors de la jurisprudence Mayotte. Les dispositions générales du texte de révision ont donc le mérite d'étendre son champ d'action référendaire.

L'idée de donner la parole aux assemblées locales et de consulter la population ne puise pas seulement sa source dans les règles communes. On la trouve aussi dans des dispositions spécifiques à l'outre-mer ${ }^{39}$. Il s'agit des articles 72-4 et 73. Seul le premier s'applique à l'ensemble de l'outre-mer ou presque $^{40}$. Le deuxième vise uniquement les départements et régions ultramarins. Dans les deux cas, une consultation est organisée afin de recueillir le consentement des électeurs. Nous nous bornerons à développer ici ce qui constitue à la fois le dernier apport commun à l'outre-mer et une

\footnotetext{
${ }^{37}$ op. cit.., p. 540.

${ }^{38}$ Voir les deux décisions du Conseil constitutionnel : $\mathrm{n}^{\circ}$ 91-290 DC, 9 mai 1991 sur la Corse et $\mathrm{n}^{\circ}$ 2000-428 DC précitée notamment le Cons. 6 ( $2^{\mathrm{e}}$ partie).

${ }^{39}$ Les dispositions des articles $72-4$ et 73 n'intéressent que l'outre-mer.

${ }^{40} \mathrm{Il}$ s'agit des départements et des régions d'outre-mer régis par l'article 73 et des collectivités d'outre-mer concernées par l'article 74.
} 
des grandes innovations de cette révision, à savoir la possibilité de changement de statut constitutionnel, par le biais d'une loi organique.

On sait que dans ce domaine, les liens du «corset juridique » ont toujours été extrêmement tendus. Il suffit de se remémorer la singularité ${ }^{41}$ de l'accord du 27 janvier 2000 entre le gouvernement et une partie de la classe politique mahoraise ${ }^{42}$ pour passer d'un statut à un autre ainsi que l'absence d'alternative donnée ${ }^{43}$ à la population pour non pas consentir mais suivre la ligne directrice. Ou encore l'exemple de Saint-Pierre-et-Miquelon où la possibilité de choix donnée à la population fut totalement vidée de son contenu par la loi qui suivit, laquelle consacra une option autre ${ }^{44}$.

L'article 72-4 qui réglemente le changement de régime constitutionnel $^{45}$, de l'article 73 vers l'article 74 ou vice versa, donne incontestablement plus de souplesse à l'évolution statutaire ultramarine. D'une part, elle confirme le cheminement parcouru depuis 2000 qui met fin au monopole gouvernemental dans le droit d'initiative ${ }^{46}$. François Luchaire souligne à juste titre l'intérêt de cette évolution ${ }^{47}$. D'autre part, elle fait du consentement des électeurs un passage «essentiel» au poids «décisif». Essentiel car passer d'un régime d'assimilation à un régime de spécialité législative ne peut pas être le fruit d'une «dérive statutaire ${ }^{48}$. Décisif car

\footnotetext{
${ }^{41}$ « Singulier » car l'accord a été conclu entre le gouvernement et des élus locaux sélectionnés et de surcroît inamendable du fait de sa publication en deux temps trois mesures dans la série Lois et Décrets du Journal Officiel.

${ }^{42}$ Le Mouvement Populaire Mahorais (M.P.M.), le Rassemblement Pour la République (R.P.R.) et le Parti Socialiste (P.S.).

${ }^{43}$ Le contenu de la question posée n'offrait pas d'alternative autre que le choix de «Collectivité Départementale».

${ }^{44}$ Le T.O.M. de Saint-Pierre-et-Miquelon a été transformé en D.O.M. en 1976 contre l'opinion de la population et contre l'avis de l'assemblée du territoire.

${ }^{45}$ Sur la procédure décrite en trois étapes, voir Thiellay, op. cit., p. 566.

${ }^{46}$ La loi d'orientation pour l'outre-mer du 13 décembre 2000 avait donné la possibilité aux conseils généraux et aux conseils régionaux d'outre-mer de se réunir en «congrès » afin de délibérer entre autres de toute proposition d'évolution institutionnelle.

${ }^{47}$ Voir Luchaire (F), «L'outre-mer devant le Conseil constitutionnel (décision $\mathrm{n}^{\circ}$ 2003-474 DC du 17 juillet 2003)», Revue du droit public, $\mathrm{n}^{\circ} 6-2003$, conclusion de la note de jurisprudence constitutionnelle.

${ }^{48}$ Comme l'a rappelée la ministre de l'outre-mer : «le président de la République s'est solennellement engagé à faire enfin inclure dans notre Constitution des garanties démocratiques incontestables pour éviter des dérives statutaires non souhaitées par nos compatriotes d'outre-mer»; J.O. Sénat, p. 3497.
} 
même si le législateur continue à rester libre ${ }^{49}$ de suivre ou pas le vote favorable à l'évolution, une chose est désormais acquise, il ne peut plus passer outre un refus considéré comme un obstacle constitutionnel ${ }^{50}$. Mayotte peut donc dormir tranquille et les forces politiques de droite de La Réunion tout autant.

Les dispositions visant l'indivisibilité de l'exercice de la souveraineté nationale ne sont pas aussi novatrices. Celles communes à l'ensemble des collectivités territoriales et celles propres aux outre-mers ont même pour point commun de ne pas remettre en cause le régime administratif antérieur. L'article 72 a surtout le mérite d'officialiser l'existence d'un pouvoir réglementaire local. Mais ce dernier n'a qu'une portée symbolique ${ }^{51}$. Conformément à la logique de l'Etat unitaire, il s'inscrit dans un cadre subordonné et respectueux des normes nationales supérieures. Même dans le cadre de l'expérimentation, ce pouvoir ne peut déroger aux dispositions du pouvoir réglementaire national sans l'accord de ce dernier et sans passer dans les fils serrés du corset ${ }^{52}$. Par ailleurs, le texte constitutionnel manque de précision car on ne sait pas exactement quel est son champ d'action.

L'article 72-2 n'est pas plus précis dans sa démarche tendant à reconnaître l'autonomie financière des collectivités territoriales. Le texte peine à donner à ce principe une définition apte à produire des effets normatifs, ce qui rend la constitutionnalisation des finances locales ambigue $^{53}$ en métropole, comme - et encore plus ${ }^{54}$ - en outre-mer.

\footnotetext{
${ }^{49}$ Sur le fait que la population ne peut en aucune manière formuler autre chose qu'un avis qui ne pourrait lier la souveraineté nationale, voir l'opinion de Michel Charasse, J.O. Sénat, p. 3495 et 3498.

50 Voir le discours de Madame Brigitte Girardin au Parlement français au sujet des référendums locaux en outre-mer : "sans cette autorisation, les pouvoirs publics ne peuvent agir plus avant, et une loi qui irait à l'encontre de la volonté populaire serait inconstitutionnelle».

51 Voir Frier (P-L), «Le pouvoir réglementaire local : force de frappe ou puissance symbolique ?» AJDA précitée, p. 563.

${ }_{52}$ Voir le contenu de l'alinéa 3 de l'article 72 qui reprend la jurisprudence du Conseil constitutionnel ; voir Brisson (J-F), «Les nouvelles clefs constitutionnelles de répartition matérielle des compétences entre l'Etat et les collectivités locales », AJDA précitée, p. 534-537.

${ }^{53}$ Hertzog $(\mathrm{R})$, «L'ambiguë constitutionnalisation des finances locales »; AJDA précitée, p. $552-554$
} 
Quant aux dispositions ultramarines, leur vocation au particularisme qui se traduit par une extension de la faculté d'interférence des collectivités territoriales d'outre-mer dans le domaine de la loi - est louable. Citons notamment celles dont bénéficient les C.O.M. qui héritent des dispositions de l'ancienne rédaction propres à la Nouvelle-Calédonie (art. 74). Ou encore celles reconnues aux départements et aux régions d'outre-mer soit pour autoadapter le droit de l'Etat dans leurs compétences (art. 73, al. 2 et 6), soit pour déroger au droit de l'Etat dans ses compétences (art. 73, al. 3 et 6) à l'exception du département et de la région de La Réunion (art. 73, al. 5).

Cette orientation ne les amène pas pour autant sur les voies d'un desserrement progressif. D'une part, parce que les normes produites restent ce qu'elles sont, de simples actes administratifs ${ }^{55}$. Seules les lois du pays de la Nouvelle-Calédonie peuvent, et ce n'est pas nouveau, prétendre à un statut quasi-législatif $^{56}$. D' autre part, parce que les liens du « corset juridique » que constituent les divers contrôles normatifs de l'Etat sont maintenus, voire renforcés $^{57}$. Olivier Gohin, dans l'article précité, relève de prime abord une constitutionnalisation de certains des termes relatifs à la déconcentration unitaire. Ainsi, le « délégué du gouvernement » devient le «représentant de l'Etat». Par ailleurs, le renforcement du contrôle normatif se justifie par la logique de la mission constitutionnelle de ce dernier, maintenue. Les normes pouvant être produites par les collectivités territoriales étant plus nombreuses et spécifiques, son rôle s'en trouve plus diversifié.

Le dernier fruit de cette manifestation persistante de l'indivisibilité est le réajustement du contenu de l'alinéa 2 du Préambule de la Constitution de 1958 qui a laissé une partie de la doctrine et même le Conseil constitutionnel $^{58}$ dans la perplexité. L'article $72-3$ alinéa $1^{59}$ consacre une

\footnotetext{
${ }^{54}$ Il y a plus de particularités au sein de l'outre-mer car elle comporte des collectivités comprenant encore de fortes spécificités par rapport aux départements de métropole. Par exemple, Mayotte.

${ }^{55}$ L'article 72 alinéa 6 maintient l'adjectif de «contrôle administratif» pour qualifier le contrôle normatif du représentant de l'Etat dans les collectivités territoriales de la République. ${ }^{56}$ La Constitution reconduit principalement les dispositions du Titre XIII à l'art. 72-3 al. 3 et ne fait aucunement mention des lois du pays. Selon les bases juridiques, à défaut d'être censurées par le Conseil constitutionnel, le Congrès vote les normes qui ne peuvent plus être contestées juridiquement ; Faberon (J.Y.), «La Nouvelle-Calédonie : vivre l'accord de Nouméa », Revue française d'administration publique, $\mathrm{n}^{\circ} 101$, janvier - février 2002, p. 46-47.

${ }^{57}$ Voir Gohin (o), art. cité, p. 527-528.

${ }^{58}$ Par exemple voir $\mathrm{n}^{\circ} 2000-428$ DC précitée ; $\mathrm{N}^{\circ} 2000$ - 435 DC, 7 décembre 2000, Loi d'orientation pour l'outre-mer; Rec Dalloz, 7 juin 2001, p. 1764 et 1765.
} 
bonne fois pour toute l'unicité des peuples français et des peuples d'outremer et cela malgré toute la diversité de ces derniers.

Ainsi le texte constitutionnel privilégie l'outre-mer en conjuguant le principe d'indivisibilité au temps de la diversité des attentes locales. Il en résulte une variabilité des statuts et régimes juridiques.

\section{B - UNE NOUVELLE CLASSIFIACTION ULTRAMARINE PLUS SOUPLE}

La révision n'a pas seulement pour effet d'ébranler la division tripartite initiale, elle entame aussi, certes relativement, les contours de la dualité traditionnelle. Commençons par relever que la catégorie des D.O.M./R.O.M. est la première bénéficiaire d'une série de changements qui lui permettent de ne plus s'aligner aussi rigoureusement sur le régime législatif métropolitain. Les nouveautés viennent d'une part contredire la jurisprudence du Conseil constitutionnel d'autre part esquisser un droit spécifique à chaque D.O.M.

Jusqu'alors en effet, la division tripartite initiale et le Conseil constitutionnel avaient un effet restrictif quant aux capacités des D.O.M./R.O.M. à s'écarter de la loi ${ }^{60}$. Les seules mesures d'adaptation qu'il était possible d'envisager devaient être nécessitées pour ces dernières par leur situation particulière et avoir une portée limitée. L'article 73 commence par étendre le champ d'application de la mesure aux actes normatifs ${ }^{61}$. Puis les adaptations possibles doivent être liées "aux caractéristiques et contraintes particulières de ces collectivités ». Ainsi les composantes de la catégorie concernée pourront non seulement déroger plus facilement à la législation ou à la réglementation nationale mais elles pourront le faire, tout comme les autres R.U.P. (Régions Ultra-Périphériques) ${ }^{62}$, sur la base de leurs caractéristiques et handicaps physiques et permanents ${ }^{63}$.

\footnotetext{
59 «La République reconnaît, au sein du peuple français, les populations d'outre-mer, dans un idéal commun de liberté, d'égalité et de fraternité ».

${ }^{60} C f$. Cons.const. 2 décembre 1982, n $82-147$ DC, R.J.C., p. 70 ; Cons. const. 25 juillet 1984, R.J.C. p. 48 ; Cons. const. 7 décembre 2000, préc.

${ }^{61}$ L'article 73 alinéa 1 fait référence aussi bien aux lois qu'aux règlements.

${ }^{62}$ Les régions ultrapériphériques mentionnées dans le traité d'Amsterdam comprennent quatre françaises : la Guadeloupe, la Guyane, la Martinique et La Réunion. Ces dernières ne sont pas
} 
De plus, et toujours contrairement à une jurisprudence établie, le dernier alinéa de l'article 73 ouvre la possibilité de créer " une collectivité se substituant à un département et une région d'outre-mer ou l'institution d'une assemblée délibérante unique pour ces deux collectivités». Ce dernier cas de figure également soumis au veto populaire ${ }^{64}$ a déjà été projeté dans les caraïbes pour remédier à l'enchevêtrement des compétences résultant de la coexistence de leurs assemblées locales. Face à des compétences ciblées, réunies au sein d'une collectivité unique, le citoyen, de Guadeloupe ou de Martinique, aurait pu mieux contrôler l'action politique, et ceci au bénéfice de la démocratie. Mais c'était sans compter avec la volonté de certains élus locaux à donner à ce simple réajustement une dimension juridico-politique plus grande.

Par ailleurs, la première catégorie du binôme offre au droit ultramarin deux enrichissements supplémentaires qui mettent en exergue l'initiative des élus locaux. Ils peuvent prévoir eux-mêmes les adaptations par rapport au droit commun, non seulement dans le cadre de matières propres à leur collectivité et des habilitations législatives (alinéa 2 dudit article), mais aussi, en vue d'une meilleure prise en compte de leurs spécificités, dans certains domaines de compétence étatique (alinéa 3). Ces ouvertures ne laissent pas de marbre, certains y voient même les premiers pas d'un risque de dérive vers l'autonomie juridique ${ }^{65}$.

C'est pourquoi des précautions ont été prises par la loi constitutionnelle du 28 mars 2003 pour remplacer les liens usés du corset. L'article 73 énonce en effet une liste non exhaustive de matières régaliennes qui ne permettent pas l'auto-adaptation du droit applicable ${ }^{66}$. Puis il laisse

désignées nominativement mais incluses sous l'appellation «les départements et régions d'outre-mer».

${ }^{63}$ Ceux-ci tiennent par exemple à leur insularité et à leur assiette territoriale très faible, à leurs conditions climatiques ou à leur isolement géographique, ainsi qu'à leur très grand éloignement par rapport au continent européen où se trouvent les principaux centres de décisions, à l'étroitesse de leur marché économique ou encore à leur dépendance parfois excessive par rapport à certains secteurs d'activité. Oraison (A), art. précité, p. 246. Sur la Guyane et ses spécificités rendant difficile l'application du droit commun, v. M. Elfort, « De la décentralisation à l'autonomie : la Guyane », Revue française d'administration publique précitée, p. 27-29.

${ }^{64} \mathrm{Le}$ consentement s'inscrit dans les mêmes formes que celui prévu au second alinéa de l'art. $72-4 \mathrm{C}$.

${ }^{65}$ Voir opinion de J.-P. Thiellay dans art. précit. p. 568.

${ }^{66} \mathrm{Cf}$. alinéa 4 de l'article cité. 
entre les mains de la collectivité concernée ${ }^{67}$ le seul monopole de la demande, sous réserve de l'existence d'une «spécificité locale», éventuellement «appréciée par le Conseil constitutionnel » ${ }^{68}$. Enfin, il interdit toute atteinte aux «conditions essentielles d'exercice d'une liberté publique ou d'un droit constitutionnel garanti $»^{69}$.

Ce qui est sûr, c'est que l'assimilation législative n'est plus ce qu'elle était. Elle s'ouvre à l'organisation particulière, un trait qui appartenait jusqu'alors à la spécialité législative. De plus, cette nouvelle orientation ne fait pas l'unanimité parmi les DOM. Les ouvertures de l'article 73 ont effrayé les élus réunionnais appartenant à la droite politique, les liens du «corset juridique » ne leur semblant pas - à l'évidence - assez solides pour résister au temps qui court. Leur refus de quitter l'embarcation traditionnelle s'est concrétisé par voie d'amendement ${ }^{70}$ et vise à un calquage exclusif de tout ce qui est consenti communément à toute collectivité territoriale, aussi bien métropolitaine qu'ultramarine, ou presque ${ }^{71}$. Cette obstination vaut à ce DOM non seulement le mérite involontaire d'approfondir la différenciation au sein de sa catégorie, mais aussi le désagrément de constituer un verrou de plus au corset et donc de ralentir la vitesse des navires de ses diverses équipières déjà emportées par les vents de la modernité.

De son côté, la catégorie des C.O.M. suit également une vocation différente de celle des T.O.M. ${ }^{72}$. Elle n'hérite pas uniquement des règles les

${ }^{67} \mathrm{Cf}$. alinéa 6 de l'article cité.

${ }^{68}$ Ces deux réserves constituent deux des cinq «verrous » énoncés par A. Oraison, v. art. précit., p. 247-248.

${ }^{69} \mathrm{Cf}$. alinéa 6 de l'article cité.

${ }^{70}$ L'amendement Virapoullé adopté par le Sénat, le 6 novembre 2002.

${ }^{71}$ L'article 73 alinéa 5 empêche La Réunion de bénéficier des alinéas 3 et 4 , c'est-à-dire des dispositions propres aux D.O.M. jugées trop audacieuses. La référence expresse au «département et à la région de La Réunion » est une manière fort habile d'échapper par ricochet non seulement à l'alinéa 6 de l'article 73, mais aussi aux dispositions communes telles que l'art. 72-4; voir Thiellay (J.P.), art. précité, p. 567.

${ }^{72}$ Voir G. Bélorgey, «Le ministère de l'outre-mer : les raisons de la permanence et les besoins de réforme ", Revue française d'administration publique précitée, p. 94-95. Selon l'auteur, le modèle des T.O.M. a toujours consisté à faire valoir les différences, d'abord pour imposer l'intérêt des dominants, ensuite pour respecter les droits et héritages des premiers habitants. Chacun des pays composant l'ancien ensemble des T.O.M., à l'exception de SaintPierre-et-Miquelon et d'une certaine manière, de Mayotte, s'est construit sur des volontés successives d'organiser des différences. La traduction juridique de ces bases historiques a été 
régissant. Doivent aussi entrer au crédit de cette nouvelle partie du binôme celles propres aux ex-collectivités sui generis. Ce qui explique la nouvelle orientation suivie par le principe de spécialité législative qui à défaut de ne plus pouvoir s'imposer comme avant devient modulable. Notons que cette nouvelle classification conduit même à un dédoublement du régime, la réécriture vise incontestablement deux catégories de collectivités distinctes, surtout quant à leur finalité juridique.

Les premiers alinéas de l'article 74 commencent par déterminer quelques règles communes dont la portée présente et à venir dépend de la loi organique. C'est cette dernière en effet qui définit le statut à la carte de chaque C.O.M. comme peut déjà l'illustrer l'exemple de la Polynésie française ${ }^{73}$. Ce statut est chargé d'abord de déterminer les compétences respectives de chacune des collectivités concernées sous réserve de ne pas contrarier une des nombreuses matières régaliennes mentionnées à l'article $73^{74}$, à moins qu'il s'agisse d'une compétence déjà attribuée ${ }^{75}$.

En outre, c'est à lui de poser les règles d'organisation et de fonctionnement des institutions ainsi que les conditions dans lesquelles ces dernières seront consultées. Ainsi, l'article 5 de la loi organique du 27 février 2004 relative à la Polynésie française fait du président la première institution de la collectivité ${ }^{76}$. Cependant, ce reclassement ne contrarie pas le principe de libre administration des collectivités territoriales par des conseils élus, car cet exécutif demeure, tout comme le gouvernement, responsable devant l'assemblée élue ${ }^{77}$. Les membres de cette dernière jouissent d'un mode de désignation spécifique qui avantage les formations les plus importantes. Il s'agit en effet du scrutin de liste à un tour applicable dans chaque circonscription. L'originalité résulte de l'attribution d'une prime majoritaire

le principe de spécialité législative tandis que, s'agissant des organes, la recherche d'institutions sur mesure a toujours été à l'œuvre.

${ }^{73}$ Loi organique $\mathrm{n}^{\circ}$ 2004-192 du 27 février 2004 portant statut d'autonomie de la Polynésie française, JORF, 2 mars 2004, p. 4183.

${ }^{74}$ Mentionnées dans le quatrième alinéa de l'article 73 de la Constitution puis complétées par l'art. 74 de la loi organique précitée.

${ }^{75}$ Le quatrième alinéa de l'article 74 réserve les compétences actuellement exercées par les collectivités concernées qu'il ne sera pas possible de remettre en cause.

${ }^{76}$ F. Luchaire, «La Polynésie française devant le Conseil constitutionnel (Décision $n^{\circ} 2004-$ 490 DC du 12 février 2004) », Revue du droit public, n6-2004, p. 1729.

${ }^{77}$ Voir Revue du droit public n ${ }^{\circ} 6-2004$ précitée, p. 1730. 
pour la liste arrivée en tête. Une fois cette attribution opérée, les autres sièges sont répartis à la représentation proportionnelle à la plus forte moyenne ${ }^{78}$.

Enfin et surtout, il doit fixer les modalités de l'application des normes nationales. En Polynésie française, on constate que la loi organique fait preuve d'une grande précision dans cette démarche car elle nous donne par exemple une énumération détaillée des domaines dans lesquels les dispositions législatives et réglementaires sont applicables de plein droit ${ }^{79}$. Même si le Conseil constitutionnel estime que celle-ci n'est pas limitative ${ }^{80}$. S'ajoute également à son mérite une contribution juridique certaine pour son rôle dans le renforcement des institutions polynésiennes ${ }^{81}$. Reste à espérer qu'elle saura aussi faire preuve de clairvoyance pour les deux anciennes collectivités sui generis régies pour partie par le principe d'identité législative, Saint-Pierre-et-Miquelon et Mayotte ${ }^{82}$.

Observons à ce stade le parcours quasiment inversé suivi par les deux catégories du nouveau binôme. Alors que dans la première, on passe de l'uniformité au particularisme, dans la seconde, on tente de sortir du particularisme pour... une certaine uniformité. Il en est de même, s'agissant de la prise en compte des aspirations locales. Dans la première, elle s'exprime par la concession de régimes juridiques différenciés alors que dans la seconde, la nouvelle rédaction tente de contenir la percée irréductible des régimes particuliers d'antan ${ }^{83}$. Pour autant, la différenciation au sein de cette dernière est toujours à l'ordre du jour comme le confirme la seconde série d'alinéas de l'article 74.

Les alinéas 7 et 11 instituent en effet une gradation de taille au sein des C.O.M. car ils réservent aux «collectivités dotées de l'autonomie » des mesures particulièrement innovantes conjuguant responsabilité locale et

\footnotetext{
${ }^{78}$ J.-E. Schoettl, «Un nouveau statut pour la Polynésie française après la révision constitutionnelle du 28 mars $2003 »$, Revue française de droit administratif, mars-avril 2004, p. 258.

${ }^{79}$ Article 7 de la loi organique précitée.

${ }^{80}$ Voir le considérant 18 de la décision $n^{\circ} 2004-490$ DC in JORF précité.

${ }^{81}$ Voir Oraison (A), « Réflexions sur la loi organique du 27 février 2004 portant statut d'autonomie de la Polynésie française (Le premier statut «cousu-main » conféré à une collectivité d'outre-mer du Pacifique en application de l'article 74 de la Constitution, revu et corrigé par la constitutionnelle du 28 mars 2003), Revue Juridique et Politique, 2004 - n 2, p. 211-229.

${ }^{82}$ Elle impliquera une réécriture potentiellement lourde de conséquences ; voir art. de J.P. Thiellay précité, p. 569.

${ }^{83}$ Voir art. précité de J.Jorda, p. 714.
} 
engagement de l'Etat. La Polynésie française étant à ce jour la seule concernée (sachant que la Nouvelle-Calédonie est classée hors catégorie), c'est donc elle qui tire avantage des mesures constitutionnelles suivantes dont la portée lui est déjà profitable.

En premier lieu, les assemblées délibérantes des collectivités territoriales de cette catégorie pourront, une fois que la loi organique statutaire sera à même d'en préciser le cadre, adopter des actes intervenant en matière législative qui resteront des actes administratifs entrant dans la compétence du Conseil d'Etat statuant au contentieux. Le terrain apparaît déjà clair quant à la faculté reconduite pour l'assemblée territoriale de légiférer dans un nombre de matières relevant $\mathrm{du}$ domaine législatif ${ }^{84}$, quant à la valeur également reconduite de ces futures normes ${ }^{85}$ et quant à la volonté de leur réserver cette fois un contrôle adapté à leur spécificité ${ }^{86}$. La loi organique du 27 février 2004 aurait alors pu donner à ces actes subordonnés une dénomination autre que «Lois du pays » car celle-ci porte à confusion ${ }^{87}$.

En deuxième lieu, le Conseil constitutionnel pourra, sur initiative de l'assemblée délibérante, déclarer une loi inconstitutionnelle dès lors qu'elle est intervenue dans un domaine de compétence d'une C.O.M. La mesure révèle une volonté manifeste du Constituant de permettre à ces collectivités d'entrer dans le cadre d'une autonomie renforcée. Cependant, nous savons qu'elle ne pourra se rapprocher du modèle dont elle s'inspire ${ }^{88}$ sans une délimitation précise des compétences territoriales. Ne reste plus qu'à la Haute Juridiction à se tenir prête à développer une jurisprudence qui ne manquera pas d'être intéressante ${ }^{89}$.

\footnotetext{
${ }^{84}$ C.C., décision $\mathrm{n}^{\circ}$ 65-34 L du 2 juillet 1965, Rec., p. 75.

${ }^{85}$ Le caractère de ces actes résulte de la jurisprudence administrative, C.E., 27 février 1970, Saïd Ali Tourqui, Lebon p. 138 ; Confirmée depuis notamment en 1992 et 2002.

${ }^{86}$ Cela correspond à la volonté locale de voir le contrôle de ces actes échapper à la compétence des tribunaux administratifs, juges de droit commun du contentieux administratif. C'est le Conseil d'Etat qui se prononce alors, par voie d'action, de manière définitive, sur leur conformité au regard de la Constitution, des lois organiques, des engagements internationaux et des principes généraux du droit ; Art. 176 et suivants de la loi organique précitée.

${ }^{87}$ On pense obligatoirement aux lois du pays de Nouvelle-Calédonie qui n'ont pas la même valeur juridique. Pour André Oraison, il s'agit d'un «abus de langage », voir art. précité sur ses réflexions concernant la loi organique du 27 février 2004, p. 223.

${ }^{88}$ Deuxième alinéa de l'art. $37 \mathrm{C}$.

${ }^{89} \mathrm{Si}$ on tient compte du fait que certains sujets lourds ont déjà fait l'objet de nombreuses frictions entre certains T.O.M. et l'Etat.
} 
En troisième lieu, et c'est ici que la Constitution suscite le plus d'interrogations, les C.O.M. dotées de l'autonomie pourront mettre en œuvre des mesures de discriminations positives en faveur de leur population, en matière "d'accès à l'emploi, de droit d'établissement pour l'exercice d'une activité professionnelle ou de protection du patrimoine foncier ». Certes, la formule n'est pas nouvelle mais elle était jusqu'alors réservée au cas particulier de la Nouvelle-Calédonie. L'utiliser à nouveau confirme un changement de philosophie et une évolution des critères utilisés qui heurtent pourtant de plein fouet des principes forts ${ }^{90}$. La loi organique du 24 février 2004 portant statut de la Polynésie française en fait en tout cas une première application sur son territoire en fondant ses mesures spécifiques ${ }^{91}$ sur des critères de résidence ${ }^{92}$.

En dernier lieu, la Constitution prévoit la participation de la collectivité à l'exercice des compétences que conservera l'Etat, "dans le respect des garanties accordées sur l'ensemble du territoire national pour l'exercice des libertés publiques». La loi organique du 27 février 2004 a le mérite de déterminer avec clarté cinq catégories de compétence ouvertes sur habilitation aux institutions de la Polynésie française ${ }^{93}$.

Quant à l'engagement de l'Etat, il est toujours de rigueur. Plus l'autonomie juridique gagne du terrain notamment dans les matières régaliennes, plus l'Etat réitère son encadrement rigide et ses garde-fous ${ }^{94}$. Au plus grand préjudice des velléités indépendantistes ${ }^{95}$.

\footnotetext{
${ }^{90}$ Notamment le principe d'égalité. Le Conseil constitutionnel admet cette incise sous réserve du respect de l'alinéa 10 de l'art. 74 C ; voir décision précit. 2004.

91 A savoir la sauvegarde du patrimoine foncier et la protection de l'emploi local dès lors toutefois qu'elles s'inscrivent dans le champ des «nécessités locales »; Art. 74 alinéa 10.

${ }^{92}$ Le critère étant la durée de résidence et des conditions étant posées comme l'égalité des mérites et la justification de la «discrimination» par des critères objectifs tenant à la nécessité de soutenir l'emploi local (alinéa 18 de la loi organique précitée). La loi du pays sera chargée de fixer la durée.

${ }^{93}$ Art. 31 de la loi statutaire.

${ }^{94}$ Ainsi par exemple si les institutions polynésiennes peuvent édicter des normes qui sont du domaine de l'Etat, au moyen d'une loi du pays, leur entrée en vigueur est subordonnée à l'accord préalable de l'Etat par voie d'un décret et ensuite d'une loi votée par le Parlement ; Art. 32 de la loi statutaire.

${ }^{95}$ Voir l'opinion du sénateur Gaston Flosse partagée - sur ce point - par M. André Oraison dans son article sur la loi organique du 27 février 2004 ; Saux (J.L.), « Un nouveau statut pour la Polynésie française », Le Monde, jeudi 15 janvier 2004, p. 11 ; Oraison (A) précité, p. 229.
} 
Ainsi l'étude de cette nouvelle classification nous révèle une réorientation des principes d'identité et de spécialité législatives dont les éléments divergents commencent à devenir de plus en plus flous. Pour André Oraison, cet état de fait risque même à terme de ne plus donner intérêt à la distinction $^{96}$.

Par ailleurs, la Constitution s'attache à moderniser un acquis du droit de l'outre-mer en mettant en valeur le pouvoir d'action des autorités compétentes de la République, notamment du Gouvernement. L'article $38 \mathrm{C}$ qui a jusqu'alors permis à ce dernier de légiférer en faveur de l'outre-mer ${ }^{97}$ est la source d'inspiration du nouvel article 74-1. Pour les C.O.M. et la Nouvelle-Calédonie, il offre au Gouvernement la possibilité «d'étendre par ordonnance avec les adaptations nécessaires, les dispositions de nature législative en vigueur en métropole ». Tout comme l'article $38 \mathrm{C}$ ou presque, l'autorité gouvernementale ne peut néanmoins agir sans un certain contrôle du Parlement ${ }^{98}$. Ceci permet d'atténuer une éventuelle main-mise de celle-ci dans un domaine qui n'est pas le sien, au préjudice éventuel d'une des collectivités. Tout particulièrement à Mayotte où les spécificités locales rendent difficiles l'application du droit commun, ces ordonnances pourraient alors apporter pour les matières non encore régies par le principe d'identité législative un coup de pouce supplémentaire au processus de transition.

$\mathrm{Si}$ certains aspects de ce dispositif peuvent continuer à servir la démocratie locale, ce n'est point le cas pour d'autres, surtout s'ils visent à resserrer un peu plus les liens du corset. C'est ce que nous verrons au cours des développements suivants. Reste en effet à voir les revers de la médaille de toutes ces avancées constitutionnelles. (II)

\footnotetext{
A moins qu'il faille se rapporter à une réalité plus neutre selon laquelle compte tenu des frontières de plus en plus flous entre autonomie et indépendance, nul ne sait qui revendiquera quoi demain et qui séduira les électeurs ; Régnault (J.M.), «Autonomie ou indépendance en Polynésie française (Vrai ou faux débat ?) », Revue Juridique et Politique, J.O. 2002 p. 81-82. ${ }^{96}$ Voir art. 2003 précité., p. 255.

${ }^{97}$ Trois lois d'habilitation avaient précisément cet objectif ( ${ }^{\circ} 2001-503$ du 12 juin 2001, $\mathrm{n}^{\circ}$ 99-899 du 25 octobre 1999 et $n^{\circ} 98-145$ du 6 mars 1998).

${ }^{98}$ Le contrôle du Parlement n'est pas le même.
} 


\section{LA DEMOCRATIE LOCALE A L'EPREUVE}

La satisfaction éprouvée de ce nouveau tournant rédactionnel est quelque peu altérée par certains éléments politiques et juridiques. D'un côté, les élus locaux se montrent particulièrement divisés sur la manière de jouir des dispositions du texte constitutionnel. Nous l'illustrerons en nous basant essentiellement sur le comportement des élus de Guadeloupe, qui nous apparaît le plus significatif ${ }^{99}$. Nous n'oublierons pas l'exemple polynésien où les dissensions entre les deux principaux leaders ont généré une crise politique importante (A). De l'autre côté, c'est le Constituant lui-même qui ne peut échapper à la critique. Car l'ensemble des dispositions suscite certaines interrogations sur les bouleversements juridiques à venir (B).

\section{A - DIVISION DES ELUS SUR FOND D'INCOHERENCE RENOUVELEE}

Suite au renforcement manifeste du poids des outre-mers au sein de la charte suprême, les élus locaux concernés se montrent particulièrement divisés sur l'application effective de certaines avancées du texte. Plus que la division elle-même, ce sont les facteurs d'incohérence, d'imprévisibilité, et même d'abus de pouvoir qui mettent à mal la démocratie locale.

Commençons par parler du cas regrettable du département de $\mathrm{La}$ Réunion $^{100}$. C'est bien lui qui le premier s'est distingué par son attachement intégral au statu quo, tel qu'il a été établi par la loi de départementalisation du 19 mars 1946. Cette position est le résultat du coup de force mené par les forces politiques de droite à l'encontre du projet de loi constitutionnelle qui posait le principe des habilitations législatives au profit des D.O.M. L'homme clé est le sénateur maire de Saint-André, Jean-Paul Virapoullé (U.M.P.), qui en dépit de la ligne directrice de Jacques Chirac annoncée à Saint-Denis même exprime sa divergence par voie d'amendement. Adopté

\footnotetext{
${ }^{99} \mathrm{Du}$ point de vue aussi bien des facteurs utilisés que de la réaction des électeurs.

${ }^{100}$ Pour André Oraison, l'exception expressément prévue au département de La Réunion est « regrettable » et est loin de constituer un progrès ; voir art. précité concernant ses analyses sur l'art. 73, p. 251-253.
} 
par le Parlement ${ }^{101}$, cet amendement deviendra l'article 73 alinéa 5 privant ainsi ce département si proche de Mayotte d'une grosse partie des apports ultramarins $^{102}$. Ce voisinage avec l'île aux parfums n'est d'ailleurs pas si anodin que cela. Car il est très probable que si Mayotte venait d'être fraîchement départementalisée, elle s'inquièterait tout autant de la remise en cause traditionnelle d'un statut si attendu !

Pourtant, ce conservatisme est unanimement fustigé par l'ensemble des forces politiques de gauche qui dénoncent l'incohérence et une certaine immaturité $^{103}$. Mme Brigitte Girardin n'était pas loin de partager cette opinion dans un premier temps avant de la relativiser ${ }^{104}$.

La Corse n'est pas en reste. Bien qu'elle ne fasse pas partie des collectivités ultramarines, cette collectivité mérite qu'on s'y intéresse pour l'influence indirecte que certains lui ont prêtée dans la Caraïbe ${ }^{105}$. Longtemps, elle a été mise à l'écart de certaines avancées ultramarines que nous avons déjà précisées. C'est d'ailleurs une des nombreuses raisons qui a motivé la refonte institutionnelle, afin de remédier à l'incohérence de la gradation entre le peuple corse et les peuples d'outre-mer ${ }^{106}$. Notons que depuis la réforme, cette collectivité particulière est toujours en marge par rapport aux collectivités ultramarines. Car si ces dernières bénéficient de toutes les dispositions applicables en métropole, l'inverse n'est pas de mise. La Corse ne peut que le regretter.

\footnotetext{
${ }^{101}$ Il a été voté par le Sénat le 6 novembre 2002 par 188 voix contre 44. Cependant, après quelques réticences à l'Assemblée nationale, il faudra de nouvelles protestations de la droite réunionnaise pour l'imposer de nouveau.

${ }^{102}$ Voir art. de J.P. Thiellay, art. cit., p. 567.

${ }^{103}$ Voir art. d' A. Oraison qui nous retrace la teneur des arguments des uns et des autres, p. 251. précit.

${ }^{104}$ Voir une interview de Brigitte Girardin, Ministre de l'outre-mer. «Il faut arrêter de jouer à se faire peur », Le Quotidien de La Réunion, mercredi 16 octobre 2002, p. 9.

${ }^{105}$ Certains élus de la Caraïbe ont tenté de faire le lien. «Je pense que le «non » corse a influencé les électeurs » pouvait-on entendre au lendemain du 7 décembre 2003 ; voir les opinions de la classe politique guadeloupéenne, dans la page Spécial référendum, FranceAntilles, 8 décembre 2003, p. 4.

${ }^{106}$ F. Luchaire a rappelé combien il était regrettable de voir uniquement les populations d'outre-mer profiter, pour de simples remaniements administratifs, du champ d'application de l'appel à la libre détermination des peuples ; voir Luchaire (F), «L'outre-mer français aujourd'hui », Revue française d'administration publique précitée, p. 11.
} 
Cela dit, elle n'est pas si dépourvue dans la mesure où elle est une des premières collectivités territoriales à avoir bénéficié de la formule permettant au législateur de créer une nouvelle collectivité en lieu et place d'une ou plusieurs autres ${ }^{107}$. Mais alors que le dispositif était suffisamment huilé pour permettre au peuple corse d'émettre un avis statutaire, ce dernier n'a pas souhaité monter dans le train qui passait. Est-il passé trop vite ? En rechercher les causes peut s'avérer utile, y compris pour les futurs candidats amenés à bénéficier des dispositions spécifiques du texte de révision.

Le Professeur Jacques Robert nous y aide lorsqu'il nous rappelle combien le référendum est un instrument au maniement délicat ${ }^{108}$. Pour lui, sa réussite suppose la réunion de trois critères cumulatifs, qui ont manqué le jour du scrutin. Le premier se situe au niveau de l'importance de l'enjeu. Il n'est pas facile de mobiliser les foules. D'où l'intérêt de donner au peuple le sentiment que leur vote va être déterminant au changement. Au demeurant, les électeurs corses n'ont pas eu ce sentiment et ont exprimé leur absence de motivation par l'abstention et par un refus de à la proposition formulée ${ }^{109}$.

Le deuxième critère est la lisibilité de la question posée. Un texte constitutionnel n'est pas forcément compréhensible au premier abord par tout un chacun. Surtout lorsque la question posée à l'électeur est longue ${ }^{110}$. C'est ici que peut alors se glisser le danger car la porte est ouverte à toutes les interprétations possibles. En l'espèce, former une seule entité au lieu de deux départements distincts évoquerait-il le maintien de la Corse au sein de la République ou le contraire ? Pour cette fois, cette persistante incohérence ${ }^{111} \mathrm{n}$ 'a pas entraîné de désaccords profonds. Tout le monde, y compris les nationalistes corses, s'est efforcé de jouer le jeu face au projet gouvernemental sachant qu'il y avait matière à y gagner.

Enfin le dernier critère est la conviction de l'initiateur. Il est clair qu'on est loin des implications personnelles du Général de Gaulle. Tout au plus, Nicolas Sarkozy est celui qui a dû en tirer les leçons. Ce «manque de

\footnotetext{
${ }^{107}$ Notamment l'article 72.1 alinéa 3.

${ }^{108}$ Robert (J), « Ne manier le référendum qu'avec prudence... », Revue du droit public, $\mathrm{n}^{\circ} 4$ - 2003.

${ }^{109}$ Le «non » à recueilli $50,98 \%$ des suffrages contre $49,02 \%$ pour le « oui » et 60,52 pour les abstentionnistes.

${ }^{110}$ Plus elle est longue, plus il y aura des termes techniques.

${ }^{111} \mathrm{Il}$ y a toujours une certaine ambivalence concernant l'enjeu, préexistante à la réforme.
} 
responsabilité » ne peut que creuser plus de distance entre la population et les politiques.

A défaut d'être concluant, le référendum corse a eu au moins le mérite de souligner certaines déficiences du dispositif. Pour l'outre-mer, celles-ci concernent surtout les deux premiers critères énoncés par J. Robert. Ainsi, un effort de simplification des termes employés, une plus grande responsabilité des politiques nationales, au moins pour savoir de quoi il retourne exactement, constituent des éléments d'enseignements à tirer. En revanche, nous ne pouvons pas dire la même chose concernant le premier critère, ce qui ne discrédite en rien celui-ci. En effet, l'argument est intéressant car il est difficile d'éviter des illusions au sein de la population en ouvrant une consultation sur une base constitutionnelle mais dépourvue de toute force juridique. En l'espèce, précisons que l'article 72-1 alinéa 3 - qui constitue la base juridique du référendum en Corse - laisse au législateur toute liberté d'appréciation pour non seulement organiser la consultation locale mais aussi aller à l'encontre de l'avis émis. Cependant, nous savons que cette disposition constitutionnelle ne concerne plus les populations ultramarines qui disposent, depuis la révision et pour le même type de réajustement territorial, de la possibilité d'émettre un véritable consentement.

Attachons-nous maintenant à l'analyse des référendums locaux organisés outre-mer et plus particulièrement à celui organisé en Guadeloupe en raison de ses résultats francs et massifs. La Guadeloupe et la Martinique ont été en effet les autres bénéficiaires de consultations populaires ouvertes sur la base de dispositions constitutionnelles spécifiques, que celles-ci soient communes à l'outre-mer ${ }^{112}$ ou propres à leur catégorie ${ }^{113}$. Comme les élus guyanais n'ont pas réussi à se mettre d'accord sur le principe et exprimer ainsi une demande locale, seules, la Martinique, la Guadeloupe et de manière distincte deux communes de cette dernière, ont eu pour la première fois la possibilité d'influer officiellement et d'une manière incontournable sur leur avenir statutaire ${ }^{114}$.

En Guadeloupe et en Martinique, la question posée aux électeurs portait sur la création d'une collectivité territoriale unique se substituant au département et à la région. Dans les deux petites îles, Saint-Barthélemy et la

${ }^{112}$ Art. 72-4 C pour Saint-Barthélemy et Saint-Martin.

${ }^{113}$ Art. 73 alinéa 7 qui renvoie à l'art. 72-4 pour la Guadeloupe et la Martinique.

${ }^{114}$ La portée des ouvertures constitutionnelles a été abordée dans la partie précédente. 
moitié française de Saint-Martin, les électeurs ont eu le choix entre le maintien du statu quo ou la mutation en collectivité d'outre-mer (C.O.M.).

Si la consultation institutionnelle des deux Iles du Nord dans le cadre de l'article 74, malgré les critiques ${ }^{115}$, s'est relativement bien passée, les deux D.O.M. n'ont pas pu profiter des liens moins tendus du corset. Non seulement les enseignements de l'échec corse n'ont pas été mis à profit, mais c'est toute l'incohérence politique locale qui a rythmé la consultation de la population mahoraise du 2 juillet 2000, dont l'exemple et les limites juridico-politiques ont déjà été précisés, qui a été reproduite.

En Guadeloupe, avant le lancement du débat sur la création d'une collectivité unique se substituant au département et à la région, la population a dû subir des moments d'incertitudes quant au cadre dans lequel celle-ci devait se situer. Le statut actuel, objet d'un attachement moral vieux de plus d'un demi siècle ${ }^{116}$, risquait d'être modifié ou remis en cause. On comprend que ces débuts d'entrée en matière, qui ont animé le Congrès, instance de délibération locale en matière d'évolution institutionnelle, aient commencé à aiguiser les craintes des électeurs guadeloupéens.

Fort heureusement, les élus départementaux et régionaux se sont entendus lors du congrès du 11 octobre 2003 sur les modalités d'organisation de la nouvelle collectivité dans le cadre de l'article 73. Une fois le projet approuvé par les Guadeloupéens, la nouvelle collectivité serait administrée par une assemblée unique de 70 membres élus à la représentation proportionnelle et par un conseil exécutif issu de cette assemblée ${ }^{117}$. Il restait à convaincre la population locale.

La tâche était d'autant plus difficile que celle-ci se trouvait déjà avant même l'ouverture de la campagne confrontée à un enjeu difficile à cerner. La question posée était encore une fois trop longue et embarrassante vu la technicité des termes employés : «Approuvez-vous le projet de création en Guadeloupe d'une collectivité territoriale demeurant régie par

\footnotetext{
${ }^{115}$ Nous verrons ultérieurement celles du constitutionnaliste Guy Carcassonne.

${ }^{116}$ Rappelons que la loi de départementalisation date de 1946. A ce titre, Dominique Chattuant a souligné avec justesse que la structure mentale du guadeloupéen a été charpentée par l'assimilation. Dès lors, tout processus tendant à remettre en cause ce régime juridique et l'ordre établi ne peut être perçu que comme une catastrophe et se heurter à un repliement réactionnel ; voir art. cité dans «Les Etudes guadeloupéennes », France Antilles du 15-16 novembre 2003.

${ }^{117}$ Voir « La consultation de tous les dangers », France Antilles précité.
} 
l'article 73 de la Constitution et donc par le principe de l'identité législative avec possibilité d'adaptation, se substituant au département et à la région dans les conditions prévues par cet article ? ${ }^{118}$. Quant à la responsabilité nationale, son absence et son retrait ${ }^{119}$ volontaire suscitaient bien des interrogations quant aux futurs liens avec la République française ${ }^{120}$. Encore une fois, fallait-il y voir la simple expression de la nouvelle orientation en matière d'évolution institutionnelle ou l'esquisse d'un désengagement progressif et ultérieur de l'Etat à l'égard des outre-mers ?

Au lieu de s'efforcer de calmer le jeu en donnant à la population l'image d'une classe politique, toutes tendances confondues, partagée certes, mais cohérente et sincère dans ses interprétations, le comportement des élus tend à discréditer la proposition de changement statutaire aux yeux de la population.

Les querelles politiciennes auxquelles se mêlent parfois les élus nationaux $^{121}$, la désinformation directement axée sur la corde sensible, notamment la perte des acquis sociaux, l'absence de discours relativement uniformes sur la portée des notions inhérentes à la question, et surtout traduits dans un langage accessible à chacun, les ambitions politiques à peine dissimulées de quelques-uns, ont engendré un climat d'incohérence renouvelé et donné aux Guadeloupéens dans leur ensemble le sentiment amer de ne pas s'y retrouver. Ainsi pendant toute la campagne électorale, la population n'a eu de cesse à se demander si son consentement allait entrainer une simplification

\footnotetext{
118 Cette question a été soumise à la population lors d'un sondage exclusif France-Antilles réalisé du 20 au 28 novembre en Guadeloupe, par IPSOS Antilles, France-Antilles, $1^{\text {er }}$ décembre 2003, p. 2.

${ }^{119}$ A la différence du référendum corse, où le Premier ministre et le Président Jacques Chirac s'étaient relativement impliqués en faveur du «oui », le gouvernement choisit l'extrême prudence. Dans un discours au Parlement, Mme Girardin a bien précisé l'intention du gouvernement de ne prendre position ni en faveur du «oui », ni en faveur du «non», «Consensus au Parlement, mais l'U.D.F. vote «non », France-Antilles, 9 novembre 2003.

${ }^{120}$ F. Bayrou, Président de l'U.D.F. s'est indigné d'une phrase de Brigitte Girardin critiquant son intervention dans le débat antillais : «Que dirait-on si un Martiniquais allait dire aux Béarnais ce que doivent être leurs institutions ?» avait-elle déclaré au Parisien. Monsieur Bayrou a estimé qu'aux détours de cette phrase, « on découvre probablement l'atteinte la plus profonde et la plus considérable qu'on puisse apporter à l'idée de la République » ; FranceAntilles, $1^{\text {er }}$ décembre 2003.

${ }^{121}$ Notamment F. Bayrou dont la venue projetée dans la Caraïbe afin de faire campagne pour le «non » a été critiquée ; voir «Le P.C.G. (Parti Communiste Guadeloupéen) veut faire taire François Bayrou », France-Antilles, 18 novembre 2003, p. 2.
} 
administrative ou un changement de statut !... C'est toute l'incertitude de la question qui l'a poussée à se réfugier derrière le vieil adage : " on sait ce qu'on perd mais on ne sait pas ce qu'on trouve ».

Notons tout de même que la consultation populaire s'est déroulée dans le respect de toutes les règles. C'est d'ailleurs dans ce cadre que les partis politiques locaux ont débuté leur campagne. Dix listes ${ }^{122}$ ont en effet déposé leur candidature en préfecture dans la perspective du référendum du 7 décembre 2003. Pour constituer une liste, il était nécessaire de présenter la signature d'au moins trois élus, qu'ils soient parlementaires, conseillers régionaux ou généraux. Plus ces élus étaient nombreux, plus la liste sur laquelle ils figuraient bénéficiait d'un temps de parole conséquent sur Télé et Radio Guadeloupe. Ce qui explique les écarts entre les listes. Ainsi la liste «Objectif Guadeloupe » est intervenue deux fois plus souvent que la liste «Guadeloupe unie, socialisme et réalités » par exemple ${ }^{123}$.

C'est le camp favorable à la collectivité unique qui a réuni une grande partie d'adhérents parmi les élus dont principalement Lucette Michaux-Chevry, sénatrice, et alors, présidente de la région Guadeloupe (Objectif Guadeloupe-U.M.P.) et Jacques Gillot, président du conseil général (Guadeloupe unie, socialisme et réalités-P.S.).

L'argument le plus fréquemment utilisé était celui de la simplification administrative. Il reposait sur l'idée qu'une seule assemblée et un seul exécutif offriraient l'avantage d'un unique interlocuteur aux administrés et aux citoyens désirant solliciter la collectivité.

Le deuxième argument utilisé par les élus a été celui d'une retombée économique profitable pour la Guadeloupe. Ainsi, l'économie d'échelle réalisée aurait permis de réduire certaines dépenses inutiles ou encore -

\footnotetext{
122 Collectif pour un oui responsable et citoyen ; Front républicain pour le non ; Rassemblement pour le non ; Solidarité Guadeloupe ; Front républicain et démocratique pour le non ; Parti communiste guadeloupéen ; Objectif Guadeloupe ; Guadeloupe unie, socialisme et réalités ; Parti progressiste démocratique guadeloupéen ; Renouveau socialiste ; voir «Dix partis, dix voix avant le référendum », France-Antilles, 18 novembre 2003, p. 2.

12349 minutes et 30 secondes pour «Objectif Guadeloupe » et 18 minutes pour « Guadeloupe unie, socialisme et réalités » sur les deux heures totales de diffusion réservées à la campagne ; «Dix partis, dix voix avant le référendum », art. précité.
} 
selon la présidente de la région - "le P.I.B. aurait pu être visualisé, compte tenu des travaux de l'I.N.S.E.E., dans toute sa réalité ${ }^{124}$.

Notons que ces deux arguments auraient peut-être intéressé la population si certains élus ne s'étaient pas efforcés de dramatiser les enjeux ${ }^{125}$.

Surtout, et c'est ici que les désaccords et les confusions ont trouvé leurs points culminants, beaucoup ont vu dans cette nouvelle collectivité le moyen d'afficher plus nettement l'identité locale. Il s'agit non seulement des anciens défenseurs de l'article 74, mais aussi des partisans de l'article 73. Car même parmi ces derniers, on pouvait percevoir un certain intérêt de voir naître plus de responsabilité guadeloupéenne : «C'est le prélude à plus de responsabilité. Si ce n'est pas la panacée de la révolution, c'est un début qui permet d'avoir une autorité, une politique et un responsable ${ }^{126}$.

Le camp du «non » a certes été minoritaire, mais il n'a pas eu grand chose à faire pour convaincre la population si ce n'est de démonter un à un les arguments de modernité et de jouer la carte de la peur ${ }^{127}$. Outre le fait de renforcer l'incohérence, ce courant a été en plus générateur de désaccords profonds au sein même des partis politiques locaux.

Y a figuré notamment Victorin Lurel, maire de Vieux-Habitants (le Front républicain pour le non - P.S.) et Philippe Chaulet, maire de Bouillante (Rassemblement pour le non - U.M.P.) pour ne citer qu'eux ${ }^{128}$. Ce choix n'est

\footnotetext{
${ }^{124}$ Voir « le débat statutaire reste institutionnel », France-Antilles, 10 novembre 2003, p. 2.

${ }^{125}$ Est dénoncée une pression psychologique exercée sur la population consistant à présenter la consultation du 7 décembre comme le rendez-vous de la dernière chance ; voir opinion de Richard Nebor, conseiller municipal de Petit-Bourg, présentée à la rubrique Courrier ; FranceAntilles, 10-11 novembre 2003 ; voir « La consultation de tous les dangers » précitée où il est reproché à la présidente de la région de « focaliser certains esprits sur des problématiques qui ont tendance à personnaliser le scrutin ». En réalité, l'enjeu de la consultation se limitait à la création d'une assemblée regroupant les compétences de la région et du département.

${ }^{126} \mathrm{Si}$ on s'en tient aux propos de J. Gillot; voir «Le débat statutaire reste institutionnel » précité, p. 2.

${ }^{127}$ Voir l'opinion du maire de Baillif, Marie-Lucile Bresleau (Objectif Guadeloupe), qui avait fait campagne pour le « oui » et qui commente l'annonce des résultats : «C'est la victoire de la peur. Lorsque l'on parlait avec les gens, ils ne nous parlaient que du R.M.I., des allocations familiales, indépendance, autonomie. Je suis déçue. Pour une fois nous avions un rendez-vous avec l'histoire, nous ne l'avons pas compris. Dès le début, il y avait cette peur. Les gens qui ont fait campagne pour le «non» savaient que cet argument serait entendu...»; FranceAntilles, 8 décembre 2003, p. 4.

${ }^{128}$ Voir «Quelle classe politique pour demain », France-Antilles, 9 décembre 2003, p.2.
} 
pas dû au hasard car le premier est celui qui a apporté l'opposition politique la plus sérieuse à la présidente de la région mais qui a aussi du même coup consolidé, à l'occasion de ce référendum, l'éclatement de la gauche. Le second n'a pas été en reste dans l'opposition menée à l'encontre de Lucette MichauxChevry. Par son départ de la liste de celle-ci pour une liste plus proche de ses aspirations, il a endossé la cape du dissident de l'U.M.P.

Les opposants à la réforme ont contesté le mode de scrutin qui ne pouvait permettre une représentation de toutes les communes. Mais ils ont surtout évoqué la question de la concentration des pouvoirs entre les mains d'une seule personne, sans contre-pouvoir garantissant les droits de l'opposition ${ }^{129}$, tout en mettant en avant le danger de l'indépendance.

Enfin, le discrédit de la classe politique s'est nourri des propos de certains responsables politiques qui n'ont pas pu se résigner, même pour la bonne cause, à faire abstraction de leurs rancœurs personnelles ou de l'intérêt de leur combat politique propre. Ils ont prôné l'abstention et favorisé l'indécision et la perplexité des électeurs. On peut citer Luc Reinette, membre du bureau politique du K.L.N.G. ${ }^{130}$, une organisation proindépendantiste, décidé à attendre un cadre plus adéquat que l'article 73, et Harry Durimel des Verts Guadeloupe fermement opposés tous ceux qui étaient proches d'Objectif Guadeloupe et notamment leur présidente ${ }^{131}$.

Le résultat des urnes n'a donc pas surpris et a démontré que les garanties offertes par la Constitution révisée n'étaient pas suffisantes pour contrecarrer les arguments des tenants du statu quo. Plus de $74 \%$ des Guadeloupéens ont répondu « Non » (plus de $90 \%$ des suffrages exprimés à Bouillante). Près d'un sur deux a préféré rester chez lui le jour du scrutin ${ }^{132} \ldots$

\footnotetext{
129 A cet argument, Mme Michaux-Chevry a fait remarquer que «les maires dans leurs communes ont les pleins pouvoirs et qu'ils ne sont pas des dictateurs pour autant »; voir « Le pouvoir confisqué », France-Antilles, 10 novembre 2003.

${ }^{130}$ Voir « Le statu quo du K.L.N.G. », France-Antilles, 10 novembre 2003.

${ }^{131} \mathrm{Au}$ lendemain de la publication d'un sondage mettant en exergue le «Non », voici un extrait du commentaire formulé par Harry Durimel : «C'est un non de défiance vis-à-vis des politiques en place. Ils sont tous assimilés à Lucette Michaux-Chevry qui pèse de tout son poids dans cette campagne... »; voir « Le non en tête : les élus réagissent », France-Antilles, 2 décembre 2003.

132 Voir «Non en Guadeloupe, Oui dans les Iles du Nord», France-Antilles, Spécial référendum, 8 décembre 2003.
} 
En Martinique, les résultats également marqués par l'abstention ont été moins francs. Le «non» l'a emporté de justesse (50,48 \% contre, $49,52 \%$ pour le $\ll$ oui ${ }^{133}$ ).

Dans les deux Iles du Nord, les électeurs ont quant à eux largement approuvé le changement statutaire ${ }^{134}$. Il faut dire que les leaders politiques, toutes appartenances confondues, ont su faire ressortir un consensus politique sans lequel tout le processus mis en place par le gouvernement n'aurait pu aboutir.

Après l'exemple guadeloupéen, évoquons enfin le cas polynésien où l'incohérence politique a résulté du comportement stratégique des deux seuls camps à même d'exercer le pouvoir ${ }^{135}$. Cette fois, les élus n'ont pas cessé de se prévaloir de certaines règles du droit applicable pour conquérir le pouvoir ou s'y maintenir.

Il s'agit, d'un côté, du sénateur (UMP) Gaston Flosse incarnant l'usure et les pratiques d'un système ${ }^{136}$, mais aussi la meilleure jonction avec la République française et son principe d'autonomie renforcée. Et, de l'autre, du chef de file des indépendantistes, Oscar Temaru, incarnant le contraire même s'il se défend de toute idée d'indépendance pour le moment.

C'est l'acharnement du premier à rester au pouvoir ainsi que l'absence de neutralité du gouvernement français dans le processus électoral ${ }^{137}$ qui ont été à l'origine de la réaction populaire et de la persistance de la crise politique. Rappelons que Gaston Flosse a été détrôné de peu lors des élections territoriales du 23 mai 2004 par son rival de toujours. Son parti, le Tahoeraa Huiraatira avait manifestement fait les frais de l'attribution au mouvement dirigé par Oscar Temaru de la prime majoritaire dans l'archipel le plus peuplé de Polynésie, les îles du Vent, ainsi que de l'alliance de ce dernier avec deux partis autonomistes.

\footnotetext{
${ }^{133}$ Voir «Une défaite du oui pour un non de justesse », France-Antilles, 8 décembre 2003. Voir aussi la note de recherche de Justin Daniel dans ce numéro.

${ }^{134} 76,17 \%$ pour le « oui » à Saint-Martin et $95,51 \%$ à Saint-Barthélemy ; voir « Les Iles du Nord représentées au Sénat? », France-Antilles, 19 décembre 2003.

${ }^{135}$ Compte tenu du scrutin de liste retenu.

${ }^{136}$ Voir « 3 rapports mettent en cause la gestion de M. Flosse », Le Monde, 22 octobre 2004, p. 10.

137 Voir «Le Conseil d'Etat est invité à annuler partiellement les élections territoriales en Polynésie française », Le Monde, 10 novembre 2004, p. 9. Dès le début du processus électoral, les propos de Mme Girardin ont révélé un désir, au plus haut niveau de l'Etat, d'une inversion.
} 
C'est la faiblesse d'écart en voix entre les deux hommes qui lui a permis, peut-être un peu trop facilement, de reprendre son fauteuil de président de Polynésie et de contester l'impartialité du scrutin dans les circonscriptions favorables à son rival. Mais le retour partiel aux urnes dans les circonscriptions visées par le Conseil d'Etat n'a pas eu pour effet de désavouer l'engouement populaire pour Oscar Temaru, ni d'ailleurs d'enrayer le conflit politique. Toutefois, ce dernier a pu s'appuyer à son tour sur le droit et le mouvement de sympathie à son égard pour asseoir enfin sa légitimité. Encore faudrait-il ajouter que les systèmes juridiques applicables dans les collectivités situées outre-mer n'échappent pas à toute ambiguïté.

\section{B - LES AMBIGUITES DU DROIT APPLICABLE}

En renforçant le poids de l'outre-mer au sein de la Constitution, le Constituant n'a pu échapper lui-même à la critique. L'ensemble des dispositions, qu'elles soient communes ou spécifiques voire ciblées, ont suscité et suscitent toujours des interrogations car elles peuvent entraîner des modifications juridiques dont il n'est pas certain que les conséquences aient toujours été parfaitement mesurées. Ce sont ces possibles inconvénients que nous nous proposons d'analyser.

Le Professeur Guy Carcassonne a certes salué l'ouverture vers une plus grande diversité de statuts mais a regretté que ces capacités nouvelles n'aient pas été plus profondément pensées, plus longuement expliquées, plus longuement débattues ${ }^{138}$. Ainsi aux Antilles, une des premières retombées du texte a été l'organisation d'un «scrutin à la va-vite » qui a surtout concerné Saint-Martin et Saint-Barthélemy. Le choix ouvert à ces deux communes d'opter pour l'article 74 lui a semblé plus drastique que celui proposé pour la Guadeloupe et la Martinique. De plus, ce choix s'est fondé sur des motivations injustifiées, notamment à Saint-Martin. Les élus de cette nouvelle C.O.M. avaient en effet souligné que le consentement de la population au référendum constituait la seule solution pour préserver leur statut fiscal spécifique et améliorer plus efficacement leur gestion ${ }^{139}$.

\footnotetext{
138 Voir «On a retrouvé la chronique de Guy Carcassonne», France-Antilles, jeudi 27 novembre 2003, p. 3.

${ }^{139}$ Voir «On a voté le 7 décembre et après ? », France-Antilles, vendredi 19 décembre 2003, p. 2.
} 
Pour le constitutionnaliste, les singularités déjà permises dans le cadre précédent, compte tenu de la situation fiscale dérogatoire à SaintMartin, démontrent bien l'inexactitude de leurs propos. De plus, la partie française de l'île n'y gagnerait aucune représentation renforcée au niveau national, ne serait pas en mesure de mieux régler ses problèmes administratifs et risquerait d'aggraver ses difficultés sociales sans se trouver dotée de moyens d'action supplémentaires pour assurer son développement. Ces critiques n'ont pas paru dissuader les intéressés, si l'on s'en tient aux propos tenus encore récemment par le maire de Saint-Barthélemy. Lors d'une conférence, ce dernier explique, d'une part, que le choix de l'article 74 est fortement lié à l'histoire de l'île empreinte à la fois de l'héritage suédois et de la centralisation de l'Etat français, et d'autre part, à la volonté de se responsabiliser davantage ${ }^{140}$.

Si l'élaboration très lente de l'avant-projet de statut ${ }^{141}$ a pour le moment figé les Saint-Martinois et les Saint-Barths dans leur statut juridique antérieur, celui-ci devrait nous permettre de lever le voile sur la portée juridique effective de leurs aspirations. Notons tout de même que si on se réfère aux mises au point faites par Mme Girardin avant l'ouverture des scrutins aux Antilles ${ }^{142}$, on ne devrait pas être trop étonné de l'orientation donnée à ces futures compétences ${ }^{143}$. Dans cet état d'esprit, le pouvoir fiscal ainsi dévolu ne pourra s'exercer que dans le cadre de conventions avec l'Etat.

Pourtant, les élus de Guadeloupe et de Martinique ont eu aussi pleinement conscience des avatars de ce possible «saut dans l'inconnu ${ }^{144}$, et ce bien avant le début de la campagne des scrutins, comme en témoignent

\footnotetext{
${ }^{140}$ Conférence organisée par le Centre d'Analyse Géopolitique et Internationale (C.A.G.I.) de l'Université Antilles-Guyane, «La question statutaire après le 7 décembre 2003 », 24 février 2005.

${ }^{141} \mathrm{Au}$ lendemain du 7 décembre, aucun décret, aucune loi, aucune circulaire n'est venue officialiser la consultation.

${ }^{142}$ Voir document compte rendu intégral, sous la présidence de M. Andrien Gouteyron, débat ouvert au Sénat sur une déclaration du Gouvernement, concernant la consultation des électeurs de Guadeloupe, de Martinique, de Saint-Barthélemy et de Saint-Martin.

143 «S'agissant de Saint-Martin et de Saint-Barthélemy, les compétences normatives accordées à ces collectivités en particulier dans le domaine fiscal, ne remettront pas en cause celles que l'Etat conservera en matière de procédure pénale, de droit pénal, mais aussi de droit commercial, monétaire et financier : en aucun cas les compétences nouvelles accordées aux deux collectivités ne pourront avoir pour effet de permettre la création de «paradis fiscaux » ou de « centres off shore »; doc. précité.

${ }^{144}$ Expression employée par Guy Carcassonne rapportée dans l'art. précité.
} 
les propos du sénateur guadeloupéen, Dominique Larifla. Dès le mois d'avril 2003 , ce dernier s'adressait à la population ${ }^{145}$ pour lui expliquer combien l'article 74 changerait la donne ${ }^{146}$.

Plus directement et plus globalement, Guy Carcassonne s'est inquiété d'une certaine incohérence du texte notamment lorsque sous prétexte de permettre à l'outre-mer de réaliser une fusion, par le biais de la collectivité unique, il a abouti à des pouvoirs plus importants qu'en métropole. AnneMarie Le Pourhiet parle même de «brume constitutionnelle », à propos de la nouvelle distinction des articles 73 et $74^{147}$.

Ajoutons que la Constitution révisée constitue par elle-même un texte parfois très difficile à comprendre et souvent imprécis pouvant laisser les lecteurs, même les plus qualifiés, dans la perplexité. Citons par exemple la disposition de la loi constitutionnelle relative à l'autonomie financière des collectivités territoriales ${ }^{148}$ qui laisse entre les mains du législateur organique ou à défaut du juge constitutionnel le soin de clarifier toutes les interrogations que soulèvent certains termes de la définition donnée ${ }^{149}$. Ce manque de clarté apparaît également à la lecture de la loi organique du 27 février $2004^{150}$, sans compter les possibles conflits à venir avec le droit communautaire, et les problèmes de gestion que la fiscalisation projetée risque de générer pour

\footnotetext{
145 Par le biais du quotidien France-antilles ; «L'assemblée unique, pourquoi, comment quand », France-Antilles, samedi 12 et dimanche 13 avril 2003.

${ }^{146}$ Selon Dominique Larifla, l'article 74 modifierait leur relation avec la République. Cette spécificité érigée en système se traduirait par davantage de pouvoir local mais avec un risque : la possibilité pour le pouvoir central de ne pas appliquer les lois nationales et notamment les lois sociales, ceci au détriment de la population.

${ }^{147}$ Le Pourhiet (A-M), «Référendums aux Antilles», "Nou pa ka acheté chat an sak», Revue du droit public, $\mathrm{n}^{\circ} 3-2004$, p. 662.

${ }^{148}$ Il s'agit de l'article 72-2.

${ }^{149}$ Par exemple, la notion de catégorie de collectivité territoriale n'apparaît pas dans le texte constitutionnel. La rédaction du projet de loi organique qui tend à donner la définition la plus simple possible, n'a retenu «que trois grandes catégories de collectivités, que sont les communes, les régions et les départements avec le rattachement des collectivités d'outre-mer ou à statut particulier à l'une ou l'autre de ces catégories, selon leur nature et les compétences exercées »; Doc. Projet de loi organique, Article premier, Définition de la notion de catégorie de collectivités territoriales.

${ }^{150}$ Voir conclusion de la note de jurisprudence constitutionnelle, Luchaire (F), Revue du droit public, $\mathrm{n}^{\circ}$ 6-2004 précité.
} 
certaines collectivités d'outre-mer, notamment à Mayotte peu habituée jusqu'alors à la taxation ${ }^{151}$.

Cette complexité et ce manque de précision peuvent même s'étendre, on l'a vu, jusque dans ses applications nationales et même locales. Ainsi, après le manque de lisibilité de la question posée à la population guadeloupéenne et martiniquaise, le comportement de leurs élus en a empêché par la suite toute clarification. Pourtant, sur le terrain, la question a été maintes fois débattue ici et là, ce qui n'a pas empêché la population d'être désorientée par des discours auxquels il lui était souvent difficile de conserver une oreille attentive compte tenu la technicité des termes employés. D'où l'incompréhension exprimée par les populations locales à une semaine des scrutins ${ }^{152}$.

Par ailleurs, force est de constater aujourd'hui que l'application de la loi organique du 27 février 2004 relative à la Polynésie française a posé des problèmes. Les dix mois de «majorité incertaine » ne peuvent manquer de susciter des interrogations quant aux retombées réelles du mode de scrutin. Pour certains, l'attribution de la prime majoritaire contraste singulièrement avec le niveau peu élevé du seuil d'accès à la représentation proportionnelle ${ }^{153}$. Il reste que le recours à deux reprises par les deux protagonistes polynésiens à la motion de censure, qui demeure une mesure exceptionnelle ${ }^{154}$, ne peut que confirmer l'inadéquation du dispositif.

Interrogeons-nous maintenant sur les possibles effets pervers de certaines dispositions de la charte suprême sur le statut communautaire de certaines collectivités territoriales ultra-marines. Plus particulièrement celles qui ont déjà constitué la base d'une proposition de fusion aux Antilles, ou encore celles qui ont permis de passer d'un statut à un autre. Les modifications statutaires réalisées seraient-elles susceptibles de contrarier la position des nouveaux ou futurs bénéficiaires ultra-marins au sein de l'Union européenne ?

\footnotetext{
${ }^{151}$ L'intégration des collectivités territoriales comprenant des fortes spécificités par rapport aux départements de métropole paraît délicate. En effet, il n'existe pas d'impôt d'Etat à Mayotte, l'ensemble de la fiscalité étant versé à la collectivité départementale.

${ }^{152}$ Il ressort d'un sondage réalisé par IPSOS France-Antilles, que le niveau d'information et de compréhension des guadeloupéens a été plutôt médiocre : $68 \%$ ont jugé la compréhension difficile ; voir «Les guadeloupéens face au projet d'une collectivité territoriale unique, France-Antilles, lundi $1^{\text {er }}$ décembre 2003, p. 3.

${ }^{153}$ Voir art. précité de Jean-Eric Schoettl, p. 258.

${ }^{154}$ Voir « Trois questions à Semir al Wardi », Le Monde, 19 octobre 2004, p. 14.
} 
On ne peut répondre à ces questions sans avoir à l'esprit la distinction, en droit communautaire, entre régions ultrapériphériques de la Communauté européenne (RUP) et Pays et territoires d'outre-mer associés à la Communauté (PTOM) ${ }^{155}$.

Seuls les D.O.M. ou plus exactement aujourd'hui les D.O.M./R.O.M. et les collectivités qui pourraient être créées en application du dernier alinéa de l'article 73 ont la position de R.U.P. de l'Union Européenne (U.E.). Cette première affirmation n'a pas coulé de source dans la mesure où l'article 299 paragraphe $2 \mathrm{du}$ Traité de Rome se limitait à évoquer «les départements français d'outre-mer». Le gouvernement est intervenu très tôt pour lever le doute et préciser qu'il ne pouvait "s'agir que d'une référence géographique aux quatre départements ainsi dénommés à la date de la signature du Traité et non pas une référence juridique ou statutaire ${ }^{156}$.

Eclaircissons aussi l'hypothèse où la région et le département disparaitraient en Guadeloupe, en Guyane ou en Martinique au profit d'une collectivité unique qui choisirait de ne pas rester dans le champ de l'article 73. Un cas voisin s'est d'ailleurs déjà produit le 7 décembre 2003 lorsque les deux ex-communes de la Guadeloupe ont choisi d'opter pour le cadre de l'article 74. La réponse reste fidèle à la réglementation passée, c'est-à-dire tant que l'appartenance à la République française est maintenue, tout changement de statut interne ne peut conduire à une modification de statut par rapport à l'U.E., qu'à la suite de négociations entre les Etats membres débouchant sur la conclusion d'un accord spécifique ${ }^{157}$.

Les T.O.M. et les collectivités sui generis, appartenant aujourd'hui pour la plupart à la catégorie des C.O.M., ont quant à eux la position de P.T.O.M. Pour les situer par rapport à l'U.E., on pourrait dire qu'ils ne sont pas intégrés à la communauté mais font partie d'un Etat membre de l'Union, au demeurant la France. Alors que les principes et réglementations énoncés par les traités et établis dans les directives, règlements et décisions communautaires s'appliquent en principe à tout le territoire européen et aux R.U.P. (avec les adaptations nécessaires), ils ne s'appliquent pas aux P.T.O.M. Cette situation peut même parfois se révéler positive pour certains d'entre eux, car ils échappent ainsi à l'applicabilité des décisions de Bruxelles.

\footnotetext{
155 Voir Ziller $(\mathrm{J})$, «L'association des pays et territoires d'outre-mer à la communauté européenne », Revue française d'administration publique précitée, p. 127.

${ }^{156}$ Voir document concernant Débat sur une déclaration du Gouvernement précité.

${ }^{157}$ Voir art. J. Ziller, précité, p. 136.
} 
Dans la même logique que précédemment, toute hypothèse de changement de statut interne, notamment la transformation d'une C.O.M. en D.O.M., n'aura aucun effet automatique. Quant au cas d'une possible indépendance d'un de ces P.T.O.M., il est prévu par leur régime d'association le maintien transitoire de ce régime jusqu'à la conclusion d'un éventuel accord d'association ${ }^{158}$.

\section{CONCLUSION}

L'étude des avancées et des effets pervers du droit applicable nous aura donc fait régulièrement osciller entre l'idée d'un simple réajustement du corset juridique et celle d'une esquisse d'un desserrement progressif.

La position du gouvernement est pourtant claire. La nouvelle rédaction ne peut s'inscrire que dans le cadre d'un simple réajustement du corset juridique. Celui-ci s'est juste limité à accompagner un cheminement plus moderne, plus souple et surtout plus démocratique pour l'outre-mer. C'est néanmoins ce changement qui a encore du mal à passer aujourd'hui. N'oublions pas que la départementalisation a une histoire de même que l'autonomie. Il est difficile de faire table rase du passé, de le dissocier du présent, d'accepter qu'il soit désormais dénaturé. Dans ce contexte, on comprend que la nouvelle voie empruntée peut parfois être perçue comme le tremplin d'un désengagement de l'Etat.

Face à ces extrêmes, notre approche sera plus nuancée. La Constitution révisée réajuste en grande partie le corset juridique d'antan, mais elle jette aussi définitivement certains liens usés. L'Etat perd en effet au change. Il cède du terrain dans son droit d'initiative, les autorités compétentes de la République ne sont plus aussi maitresses de leurs normes. L'avenir nous dira jusqu' où l'Etat unitaire peut encore aller sans se remettre en cause.

${ }^{158}$ Voir art. J. Ziller, p. 136 précit. 


\begin{abstract}
RESUME
Les avancées et les revers de la révision constitutionnelle en outre-mer : simple réajustement du «corset juridique» ou esquisse d'un desserrement progressif et futur?

La révision constitutionnelle du 28 mars 2003 est une étape mémorable pour les outre-mers. En effet, les aspirations locales sont enfin valorisées dans un cadre juridique qui conjugue les dispositions relatives à l'intégralité du territoire et à l'indivisibilité de la République au temps de la diversité, de la souplesse, de la modernité. Cependant, cette nouvelle orientation appelle des craintes car elle génère des avancées décisives, notamment en matière statutaire. Et malgré les garanties offertes par le texte, celles-ci ont parfois du mal à s'estomper. Finalement, s'agit-il d'un simple réajustement du « corset juridique » ou l'esquisse d'un desserrement progressif et futur? La réponse n'est pas anodine car l'Etat unitaire français y joue son image.

Mots-clés : démocratie locale, outre mer, révision constitutionnelle, collectivité d'outre mer, territoire d'outre mer.
\end{abstract}

\begin{abstract}
The review of the Constitution and its breakthroughs and setbacks in the Overseas territories: simply a re-adjustment of the "legal corset" or the beginnings of a gradual and future letting-go?

The March 28, 2003 review of the Constitution is a memorable phase for the French Overseas territories. In fact, local aspirations are finally valorised within a legal framework which combines the mechanisms related to the unity and indivisibility of the French Republic in times of diversity, flexibility and modernity. Nevertheless, this new orientation incites fear for it generates decisive breakthroughs, especially in matters of political status. And despite the guarantees offered by the text, such fears do not easily fade. Finally, is it simply a re-adjustment of the "legal corset" or the beginnings of a gradual and future letting-go? The answer is not insignificant, for the image of a unitarian French State is at stake.

Key Words: Local democracy, overseas, Constitutional review, Overseas institutions, Overseas territory.
\end{abstract}

